\title{
The effective action of warped $M$-theory reductions with higher-derivative terms. Part II
}

\section{Thomas W. Grimm, Tom G. Pugh and Matthias Weissenbacher}

Max Planck Institute for Physics, Föhringer Ring 6, Munich, 80805 Germany

E-mail: grimm@mpp.mpg.de, pught@mpp.mpg.de, mweisse@mpp.mpg.de

ABSTRACT: We study the three-dimensional effective action obtained by reducing elevendimensional supergravity with higher-derivative terms on a background solution including a warp-factor, an eight-dimensional compact manifold, and fluxes. The dynamical fields are Kähler deformations and vectors from the M-theory three-form. We show that the potential is only induced by fluxes and the naive contributions obtained from higher-curvature terms on a Calabi-Yau background vanish once the back-reaction to the full solution is taken into account. For the resulting three-dimensional action we analyse the Kähler potential and complex coordinates and show compatibility with $\mathcal{N}=2$ supersymmetry. We argue that the higher-order result is also compatible with a no-scale condition. We find that the complex coordinates should be formulated as divisor integrals for which a non-trivial interplay between the warp-factor terms and the higher-curvature terms allow a derivation of the moduli space metric. This leads us to discuss higher-derivative corrections to the M5-brane action.

Keywords: Flux compactifications, Supergravity Models, Supersymmetric Effective Theories, M-Theory

ARXIV EPRINT: 1507.00343 


\section{Contents}

1 Introduction 1

2 Dimensional reduction of the M-theory action 3

2.1 Higher-order background solution 3

2.2 Considered variations of the background solution 5

2.3 Scalar potential 6

$\begin{array}{lll}2.4 & \text { Three-dimensional effective action } & 7\end{array}$

2.5 Warp-factor scaling symmetry and integration 9

3 Demonstrating the supersymmetric structure 11

3.1 Comparing the reduction result with $\mathcal{N}=2$ supergravity 11

$\begin{array}{ll}3.2 \text { Kähler potential and coordinates as a } \delta v \text { expansion } & 13\end{array}$

$\begin{array}{lll}3.3 & \text { Completing the Kähler potential and complex coordinates } & 15\end{array}$

$\begin{array}{llr}4 \text { Conclusions } & 19\end{array}$

\section{Introduction}

Three-dimensional effective theories arising from M-theory on eight-dimensional compact manifolds are of both conceptual as well as phenomenological interest. For example Mtheory backgrounds that admit a torus fibration allow a lift of the three-dimensional theories to four dimensions [1] that can be phenomenologically compelling. The fourdimensional theories are minimally supersymmetric admitting four supercharges if the three-dimensional effective theories are $\mathcal{N}=2$ supersymmetric. With this motivation in mind, we will study in this work a general class of M-theory reductions and argue that they are compatible with $\mathcal{N}=2$ supersymmetry. More precisely, we analyse the perturbations of warped solutions with an eight-dimensional compact internal manifold and background fluxes.

The background solutions of interest have first been considered in [2]. At leading order the background is simply a direct product of three-dimensional Minkowski space and a Calabi-Yau fourfold without background fluxes. When including background fluxes and all relevant higher-derivative terms it was shown that the internal background is conformally Kähler with vanishing first Chern class, but that the metric is non-Ricci-flat even when allowing for a conformal rescaling including the warp factor [3, 4]. A complete check of supersymmetry at this order of derivatives is still missing. However, using a proposed correction the eleven-dimensional gravitino variations based on $[5,6]$ it was shown in [4] that supersymmetry can be preserved by this background. The fluctuations of this solution were then studied in [7] and their three-dimensional effective action was derived by dimensional 
reduction. More precisely, a finite number of Kähler deformations of the metric and vector deformations of the M-theory three-form were included when reducing the leading elevendimensional supergravity action of [8] corrected by the terms fourth order in the Riemann curvature [9-15], and the higher-derivative terms quadratic in the M-theory three-form [16]. The resulting effective action was given to quadratic order in the scale parameter $\alpha \propto \ell_{M}^{3}$, where $\ell_{M}$ is the eleven-dimensional Planck length. The present work is a continuation of [7], which discusses in more detail the scalar potential and the supersymmetry properties of the three-dimensional effective theory.

In reference [7] it was shown that the number of Kähler deformations and vector zero modes are still given by the dimension of the second cohomology of the compact manifold. While the Kaluza-Klein reduction is originally no longer performed by an expansion into harmonic forms of the underlying Calabi-Yau geometry, it was shown that all corrections, associated with these alternative fluctuations, drop from the effective action. The kinetic terms for the deformations and vectors in the three-dimensional effective theory were written in terms of a single higher-curvature building block $Z_{m \bar{m} n \bar{n}}=\frac{1}{4 !}\left(\epsilon_{8} \epsilon_{8} R^{3}\right)_{m \bar{m} n \bar{n}}$, where $R$ is the internal Riemann tensor in the underlying Calabi-Yau metric. ${ }^{1}$ Furthermore, in [7] the warp-factor was fully included in the reduction. It was shown that the effective action contains integrals depending on the bare warp-factor and its first derivatives with respect to the Kähler structure deformations. Remarkably, these derivative couplings only appear through covariant derivatives under a moduli dependent scaling symmetry under which the Kähler structure deformations and the warp-factor transform. The classical leading order three-dimensional $\mathcal{N}=2$ theory obtained from M-theory on a Calabi-Yau fourfold with background fluxes was first found in [18, 19], while recent derivations of $\mathcal{N}=1$ effective theories arising from M-theory flux compactifications can be found in [20-22]. Let us note that previous works on warped compactifications of M-theory and Type IIB include [2333]. Our analysis of the $\mathcal{N}=2$ supergravity data also extends the works [34-36], which presented a partial reduction from eleven to three dimensions including some of the relevant higher-derivative terms. Recent interesting results on dimensional reductions with higher-derivative terms can also be found in [37, 38].

In a first step, and as completion of the results of [7], we derive the scalar potential for the Kähler structure deformations by dimensional reduction. Interestingly, reducing the higher-curvature terms on the leading order Calabi-Yau background it appears that they become massive with a coupling purely depending on the geometry. However, we will show that these mass terms are precisely cancelled by the higher-order corrections in the solution arising as a back-reaction effect. The remaining scalar potential is only induced by background fluxes as in [19]. This gives a further test that the included fluctuations are indeed the relevant light degrees of freedom and highlights the interplay from back-reaction effects in the solution and the corrections to the effective theory.

In order to reveal the supersymmetry properties of the three-dimensional effective action we discuss its promotion into the standard $\mathcal{N}=2$ form. In three space-time dimensions massless vectors are dual to scalars and the dynamics of the light modes therefore should be

\footnotetext{
${ }^{1}$ The equivalent quantity on a Calabi-Yau threefold was found to be important in [17].
} 
describable by a Kähler potential and a set of complex coordinates. We study the order by order expansion of the Kähler potential and complex coordinates in the Kähler structure fluctuations. The coefficients are deduced by comparison with the dimensionally reduced action. We infer compatibility with $\mathcal{N}=2$ supersymmetry and argue that a no-scale condition can be implemented. Since the dimensional reduction only includes the leading-order terms in the fluctuations we are not able to completely fix all coefficients by the comparison alone. The fundamental 'all-order' expression, as it is known for the classical reduction without higher-curvature terms $[18,19]$, turns out to be even more difficult to find. We argue that this problem lies in fixing the complex coordinates and should be approached by introducing divisor integrals. These integrals should be matched with the actions of M5-branes wrapped on divisors. We make steps towards finding an all order expression for the complex coordinates and Kähler potential. An intriguing interplay between variations of warped divisor integrals and higher-curvature terms via the warp-factor equation allows the compatibility with the dimensional reduction to be shown. As a byproduct this suggests that the M5-brane action should receive higher-curvature corrections that parametrise the non-harmonicity of the fourth Chern-form of the background geometry.

The paper is organised as follows. In section 2 we recall the background solutions, introduce an appropriate set of fluctuations, and review the dimensionally reduced effective action following [7]. In addition, we analyse the scalar potential and comment on a scaling symmetry of the effective action. The $\mathcal{N}=2$ supersymmetric structure and the noscale condition are discussed in section 3. We derive the Kähler potential and complex coordinates as an expansion in the fluctuations and later propose a definition using divisor integrals.

\section{Dimensional reduction of the M-theory action}

In this section we first review the background solution of eleven-dimensional supergravity including higher-derivative terms following $[3,4]$. We then introduce the variations of the solutions considered in [7] and show that at order $\alpha^{2}$ they only admit a scalar potential due to background fluxes. We recall the complete three-dimensional effective action including all order $\alpha^{2}$-terms following [7] and discuss its various building blocks and symmetries.

\subsection{Higher-order background solution}

To begin with we first review the warped solutions following $[3,4]$. These backgrounds satisfy the eleven-dimensional field equations to order $\alpha^{2}=\frac{\left(4 \pi \kappa_{11}^{2}\right)^{\frac{2}{3}}}{(2 \pi)^{4} 3^{2} 2^{13}}$. The eleven-dimensional metric in this background takes the form

$$
d \hat{s}^{2}=e^{\alpha^{2} \Phi}\left(e^{-2 \alpha^{2} W} \eta_{\mu \nu} d x^{\mu} d x^{\nu}+2 e^{\alpha^{2} W} \check{g}_{m \bar{n}} d y^{m} d y^{\bar{n}}\right)+\mathcal{O}\left(\alpha^{3}\right),
$$

where $\eta_{\mu \nu}$ is the three-dimensional Minkowski metric and

$$
\check{g}_{m \bar{n}}=g_{m \bar{n}}+\alpha^{2} g_{m \bar{n}}^{(2)}+\mathcal{O}\left(\alpha^{3}\right) .
$$

The internal compact manifold will be denoted by $Y_{4}$ and $\Phi$ and $W$ are scalar functions on this space. $W$ is the warp-factor and is constrained by a differential equation (2.9). For 
simplicity, and in contrast to [4, 7], we will not always indicate the $\alpha$-order at the symbol, i.e. we write $\Phi \equiv \Phi^{(2)}$ and $W \equiv W^{(2)}$. Furthermore, we will use $g_{m \bar{n}} \equiv g_{m \bar{n}}^{(0)}$ to denote the zeroth-order metric. All quantities, such as higher-curvature terms on $Y_{4}$, are always evaluated in this zeroth-order metric $g_{m \bar{n}}$ unless indicated explicitly. This will simplify the notation compared to $[4,7]$. For example, $\Phi$ represents an eleven-dimensional Weyl rescaling and is given in terms of the lowest order metric $g_{m \bar{n}}$ as

$$
\Phi=-\frac{512}{3} Z, \quad Z=*\left(J \wedge c_{3}\right),
$$

where $*$ is the Hodge-star on $Y_{4}$ in the metric $g_{m \bar{n}}, J$ is the Kähler form built from $g_{m \bar{n}}$ and $c_{3}$ is the third Chern form built from $g_{m \bar{n}}$ as

$$
c_{3}=-\frac{i}{3} \mathcal{R}_{m}{ }^{n} \wedge \mathcal{R}_{n}{ }^{r} \wedge \mathcal{R}_{r}{ }^{s} .
$$

In this expression we have used the definition of the two-form $\mathcal{R}_{m}{ }^{n}$ that is built from the Riemann tensor as $\mathcal{R}_{r}{ }^{s}=R_{m \bar{n}}{ }^{s} d y^{m} d y^{\bar{n}}$.

In order to give the expressions (2.2) and (2.3) we note that at zeroth order in $\alpha$ the background is a direct product and $g_{m \bar{n}}$ is a Ricci flat metric on a Calabi-Yau fourfold. We therefore can introduce complex indices, which here and in the following always refer to the zeroth order complex structure on the internal manifold. On a Calabi-Yau fourfold there exists a nowhere vanishing covariantly constant Kähler form $J$ and holomorphic (4,0)-form $\Omega$ satisfying

$$
d J=d \Omega=0 .
$$

In what follows we will work in conventions in which the internal space indices are raised and lowered with the lowest order internal space metric $g_{m \bar{n}}$. At second order in $\alpha$ the metric is corrected by $g_{m \bar{n}}^{(2)}$ in (2.2). This is constrained by the higher-derivative Einstein equations that are solved by

$$
g_{m \bar{n}}^{(2)}=768 \partial_{m} \bar{\partial}_{\bar{n}} \tilde{F}, \quad \tilde{F}=*\left(J \wedge J \wedge F_{4}\right) .
$$

Here $F_{4}$ is a four-form parameterising the non-harmonic part of the third Chern-from. Since $c_{3}$ is closed on a Kähler manifold we may write

$$
c_{3}=H c_{3}+i \partial \bar{\partial} F_{4},
$$

where $H$ indicates the projection to the harmonic part associated with the metric $g_{m \bar{n}}$. The expression (2.6) implies that the metric $\breve{g}_{m \bar{n}}$ introduced in (2.2) is still Kähler and that the internal part of the eleven-dimensional metric (2.1) is conformally Kähler.

The background also includes a flux for the four-form given by

$$
\begin{array}{ll}
\hat{G}_{m \bar{n} r \bar{s}}=\alpha G_{m \bar{n} r \bar{s}}+\mathcal{O}\left(\alpha^{3}\right), & \hat{G}_{m n r s}=\alpha G_{m n r s}+\mathcal{O}\left(\alpha^{3}\right), \\
\hat{G}_{\mu \nu \rho m}=\epsilon_{\mu \nu \rho} \partial_{m} e^{-3 \alpha^{2} W}+\mathcal{O}\left(\alpha^{3}\right) . &
\end{array}
$$

In order that the eleven-dimensional field equations are solved to order $\alpha^{2}$ by this background the flux $G$ must be self-dual in the lowest-order metric $g_{m \bar{n}}$. This condition allows 
$(2,2)$ and $(4,0)+(0,4)$ components of the flux with respect to the lowest order complex structure. The profile of the warp-factor $W$ depends both on the background flux $G$ and the higher-curvature terms through the equation

$$
d^{\dagger} d e^{3 \alpha^{2} W} *_{8} 1-\alpha^{2} Q_{8}+\mathcal{O}\left(\alpha^{3}\right)=0,
$$

where

$$
Q_{8}=-\frac{1}{2} G \wedge G-3^{2} 2^{13} \alpha^{2} X_{8}=-\frac{1}{2} G \wedge G+3072 c_{4} .
$$

In this expression $c_{4}$ is the fourth Chern-form evaluated in the metric $g_{m \bar{n}}$ given by

$$
c_{4}=\frac{1}{8}\left(\mathcal{R}_{m}{ }^{n} \mathcal{R}_{n}{ }^{m} \mathcal{R}_{r}{ }^{s} \mathcal{R}_{s}{ }^{r}-2 \mathcal{R}_{m}{ }^{n} \mathcal{R}_{n}{ }^{r} \mathcal{R}_{r}{ }^{s} \mathcal{R}_{s}{ }^{m}\right)
$$

Asserting that $Y_{4}$ is compact, the warp-factor equation (2.9) implies the global consistency condition

$$
\frac{1}{3^{2} 2^{14}} \int_{Y_{4}} G \wedge G=\frac{\chi\left(Y_{4}\right)}{24}
$$

where $\chi\left(Y_{4}\right)=-4 ! \int_{Y_{4}} X_{8}=\int_{Y_{4}} c_{4}$ is the Euler number of $Y_{4}$. This implies that, by using the self-duality of $G$, the higher-derivative terms cannot be consistently ignored if one allows for a background flux. ${ }^{2}$

Supersymmetry of the solution (2.1), (2.8) has not been demonstrated to order $\alpha^{2}$. This can be traced back to the fact that the supersymmetry variations of the fermions have not been derived to this order. In [4] a proposal was made for the gravitino variations including order $\alpha^{2}$ terms based on [5,6], and supersymmetry was successfully checked. Asserting that the gravitino variations, as the ones proposed in [4], are unchanged at linear order in $\alpha$, then the flux $G$ satisfies

$$
G_{m n r s}=0
$$

i.e. its $(4,0)$ component vanishes, and respects the primitivity condition

$$
G \wedge J=0 .
$$

In this work we will provide further evidence that the solution preserves supersymmetry. We derive the three-dimensional action and demonstrate compatibility with threedimensional $\mathcal{N}=2$ supersymmetry. Furthermore, we show that the scalar potential vanishes to order $\alpha^{2}$ when imposing (2.13) and (2.14) .

\subsection{Considered variations of the background solution}

Having reviewed the background solution in subsection 2.1, we now include a well-defined set of variations around this vacuum and recall the derivation of their effective action.

Firstly, we will include vectors $A^{i}$ that arise in perturbations of the M-theory threeform $\hat{C}$. These correspond to extra terms in the expansion of $\hat{G}$ of the form

$$
\delta \hat{G}=F^{i} \wedge \omega_{i}^{(\mathrm{v})},
$$

\footnotetext{
${ }^{2}$ The numerical factor in (2.12) can be attributed to our normalisation of $G$ with $\alpha$ and can be removed when moving to quantised fluxes $G^{\text {flux }}=\frac{1}{32^{6} \sqrt{2}} G$.
} 
where $F^{i}=d A^{i}$ are the field strengths of $A^{i}$, and $\omega_{i}^{(\mathrm{v})}$ are two-forms on the internal manifold. Importantly, it was argued in [7] that in the expansion $\omega_{i}^{(\mathrm{v})}=\omega_{i}^{(0)(\mathrm{v})}+\alpha^{2} \omega_{i}^{(2)(\mathrm{v})}$ only the harmonic part of $\omega_{i}^{(\mathrm{v})}$ contributes in the effective action. We may pick $\omega_{i}^{(0)(\mathrm{v})}$ to be harmonic and drop $\omega_{i}^{(2)(\mathrm{v})}$. This implies that $\omega_{i}^{(\mathrm{v})}$ can be chosen to be harmonic $(1,1)$-forms and one has $i=1, \ldots, \operatorname{dim}\left(H^{1,1}\left(Y_{4}\right)\right)$, where $H^{1,1}\left(Y_{4}\right)$ is the $(1,1)$-form cohomology of $Y_{4}$ whose dimension is independent of the metric chosen on $Y_{4}$.

Secondly, one can analyse the Kähler structure deformations of the conformally Kähler metric in (2.1). We introduce variations

$$
\delta g_{m \bar{n}}=i \delta v^{i} \omega_{i m \bar{n}}^{(\mathrm{s})},
$$

where $g_{m \bar{n}}$ is the Kähler metric given in (2.2). The $\delta v^{i}$ correspond to scalars in the threedimensional effective theory, while the $\omega_{i m \bar{n}}^{(\mathrm{s})}$ is a set of two-forms on $Y_{4}$ chosen to ensure that the Kähler condition remains to be satisfied. Remarkably, expanding $\omega_{i}^{(\mathrm{s})}=\omega_{i}^{(0)(\mathrm{s})}+\alpha^{2} \omega_{i}^{(2)(\mathrm{s})}$ it was again shown in [7] that only the harmonic part of $\omega_{i}^{(\mathrm{s})}$ contributes in the effective action. We therefore drop $\omega_{i}^{(2)(\mathrm{s})}$ and chose $\omega_{i}^{(0)(\mathrm{s})}$ to be the same harmonic $(1,1)$-forms as in $(2.15)$ with $i=1, \ldots, \operatorname{dim}\left(H^{1,1}\left(Y_{4}\right)\right)$, i.e. we set

$$
\omega_{i}^{(0)(\mathrm{s})}=\omega_{i}^{(0)(\mathrm{v})}=\omega_{i},
$$

where $\omega_{i}$ are the harmonic $(1,1)$-forms in the Ricci-flat zeroth-order metric $g_{m \bar{n}}$. In the following it turns out to be convenient to define scalars $v^{i}$ containing the background value of $g_{m \bar{n}}$ by setting

$$
g_{m \bar{n}}+\delta g_{m \bar{n}}=i v^{i} \omega_{i m \bar{n}} .
$$

When discussing Kähler structure deformations one has to carefully vary the complete background solution. In particular, all metric dependent quantities, such as the scalar function $Z$ introduced in (2.3), vary non-trivially. The second order corrections to the background turn out to be crucial when determining the mass of the fields $\delta v^{i}$. Recall that in general the primitivity condition (2.14) is not preserved by all $\delta v^{i}$. This implies that one expects a scalar potential depending on the flux $G$ as studied in the Calabi-Yau fourfold reductions with fluxes in $[18,19]$. It could, moreover, be the case that the higher-curvature terms induce additional potential terms. We will show in the next subsection that this is not the case when including $\alpha^{2}$ corrections both in the background solution and in the eleven-dimensional action.

\subsection{Scalar potential}

In this subsection we discuss the derivation of the scalar potential for the Kähler structure fluctuation $\delta v^{i}$ introduced in (2.16). As already pointed out, we expect a flux-induced scalar potential for all fluctuations that do not respect the primitivity condition (2.14).

To begin with we consider the terms containing $\hat{C}$ without derivative. Considering the pure three-dimensional space-time part for $\hat{C}$ one easily sees

$$
-\left.\int\left(\frac{1}{6} \hat{C} \wedge \hat{G} \wedge \hat{G}+3^{2} 2^{13} \hat{C} \wedge \hat{X}_{8}\right)\right|_{\text {pot }}=0
$$


which can be traced back to the fact that this combination is proportional to the tadpole constraint (2.12). A pure flux-induced potential term arises from the reduction

$$
-\left.\int \frac{1}{2} \hat{G} \wedge \hat{*} \hat{G}\right|_{\text {pot }}=-\alpha^{2} \int_{\mathcal{M}_{3}} *_{3} 1 \int_{Y_{4}} \frac{1}{2} G \wedge *^{\prime} G,
$$

where $*^{\prime}$ is the Hodge star of the perturbed internal metric (2.18). In order to derive the full flux-induced potential, however, we need to also dimensionally reduce the higher-curvature terms. Inserting the fluctuated ansatz into the $\hat{R}^{4}$-corrections to the eleven-dimensional action we find

$$
\begin{aligned}
\int \hat{t}_{8} \hat{t}_{8} \hat{R}^{4} \hat{*} 1 & =\int_{\mathcal{M}_{3}} *_{3} 1 \int_{Y_{4}}\left(1536 c_{4}-768 \delta v^{i} \delta v^{j}\left(\nabla_{a} \nabla^{a} Z\right) \omega_{i m \bar{n}} \omega_{i} \bar{n} m * 1\right) \\
-\int \frac{1}{24} \hat{\epsilon}_{11} \hat{\epsilon}_{11} \hat{R}^{4} \hat{*} 1 & =\int_{\mathcal{M}_{3}} *_{3} 1 \int_{Y_{4}} 1536 c_{4} .
\end{aligned}
$$

We thus encounter the integral over the forth Chern-form $\int_{Y_{4}} c_{4}=\chi\left(Y_{4}\right)$ and (2.12) can be used to replace these terms with a flux-dependent contribution proportional to $\int_{Y_{4}} G \wedge G$. Furthermore, there appears to be an additional mass term for the fluctuations $\delta v^{i}$ involving the higher-curvature invariant $Z$. However, we still need to dimensional reduce the zeroth order action inserting the $\alpha^{2}$-corrected background solution. Performing this reduction one finds

$$
\int \hat{R} \hat{*} 1=\alpha^{2} \int_{\mathcal{M}_{3}}{ }_{3} 1 \int_{Y_{4}} 768 \delta v^{i} \delta v^{j}\left(\nabla_{a} \nabla^{a} Z\right) \omega_{i m \bar{n}} \omega_{i}^{\bar{n} m} * 1
$$

which precisely cancels the $Z$-dependent mass-term arising from the higher-curvature reduction in (2.21).

In summary, adding all terms (2.19)-(2.22) one finds the scalar potential term

$$
S_{\mathrm{pot}}=-\frac{\alpha^{2}}{4 \kappa_{11}^{2}} \int_{\mathcal{M}_{3}} *_{3} 1 \int_{Y_{4}} \frac{1}{2}\left(G \wedge *^{\prime} G-G \wedge G\right) .
$$

This term has to be still Weyl-rescaled to bring the action into the three-dimensional Einstein frame. The rescaled result will be given in (2.26). As expected one can check that the scalar potential vanishes for primitive $(2,2)$-fluxes, i.e. for all $(2,2)$-fluxes satisfying $G_{m \bar{n} \rho \bar{s}} J^{\prime \bar{r} s}=0$. This condition generically fixes a number of deformations $\delta v^{i}$ in the vacuum. Note that this is the only effect stabilising moduli at order $\alpha^{2}$ in our setting.

\subsection{Three-dimensional effective action}

Having discussed the scalar potential, we now recall the complete three-dimensional effective action for the fluctuations $\delta v^{i}$ and vectors $A^{i}$ following [7]. It was shown in this work that it takes the remarkably simple form

$$
\kappa_{11}^{2} S_{\text {eff }}=S_{\text {kin }}+S_{\mathrm{CS}}+S_{\text {pot }}
$$

with kinetic terms given by

$$
S_{\text {kin }}=\int_{\mathcal{M}_{3}}\left(\frac{1}{2} R * 1-\frac{1}{2}\left(G_{i j}^{T}+\mathcal{V}_{T}^{-2} K_{i}^{T} K_{j}^{T}\right) D v^{i} \wedge * D v^{j}-\frac{1}{2} \mathcal{V}_{T}^{2} G_{i j}^{T} F^{i} \wedge * F^{j}\right),
$$


and flux-induced Chern-Simons terms and scalar potential given by

$$
S_{\mathrm{CS}}=\int_{\mathcal{M}_{3}} \frac{1}{2} \Theta_{i j} A^{i} \wedge F^{i}, \quad S_{\mathrm{pot}}=-\alpha^{2} \int_{\mathcal{M}_{3}} *_{3} 1 \int_{Y_{4}} \frac{1}{8 \mathcal{V}_{0}^{3}}\left(G \wedge *^{\prime} G-G \wedge G\right)
$$

The Chern-Simons terms are dependent on the fluxes via $\Theta_{i j}=\frac{\alpha}{2} \int_{Y_{4}} G \wedge \omega_{i} \wedge \omega_{j}$. In the following we introduce the remaining coefficient functions appearing in (2.24).

To begin with, as in the leading order reduction, we use the quadruple intersection numbers $K_{i j k l}=\int_{Y_{4}} \omega_{i} \wedge \omega_{j} \wedge \omega_{k} \wedge \omega_{l}$ to define

$$
\mathcal{V}=\frac{1}{4 !} K_{i j k l} v^{i} v^{j} v^{k} v^{l}, \quad K_{i}=\frac{1}{3 !} K_{i j k l} v^{j} v^{k} v^{l}, \quad K_{i j}=\frac{1}{2} K_{i j k l} v^{k} v^{l} \quad K_{i j k}=K_{i j k l} v^{l} .
$$

Here we have used the fully fluctuated $v^{i}$ defined in (2.18). In the background they take the value $v_{0}^{i}$. In this case we write, for example, $\mathcal{V}$ as $\mathcal{V}_{0}$ and $K_{i}$ as $K_{i}^{0}$. $\mathcal{V}_{0}$ is simply the background zeroth-order volume of $Y_{4}$ also given by $\mathcal{V}_{0}=\int_{Y_{4}} * 1$.

Starting with the classical couplings, one can next include the warp-factor. It turns out to be convenient to define the warped volume and the warped metric as

$$
\mathcal{V}_{W}=\int_{Y_{4}} e^{3 \alpha^{2} W} * 1, \quad G_{i j}^{W}=\frac{1}{2 \mathcal{V}_{W}} \int_{Y_{4}} e^{3 \alpha^{2} W} \omega_{i} \wedge * \omega_{j}
$$

The dimensionally reduced action also contains the first derivatives of the warp-factor with respect to the moduli $v^{i}$. They appear only through a covariant derivative

$$
D v^{i}=d v^{i}+\alpha^{2} \frac{1}{\mathcal{V}_{0}} d v^{j} v_{0}^{i} \int_{Y_{4}} \partial_{v^{j}} W * 1
$$

The significance of this fact will be discussed in detail in subsection 2.5, where we will recall the invariance of the action under a moduli-dependent scaling symmetry. Finally, in order to present to full result (2.24) we have to include the corrections due to higher-curvature terms. We first define

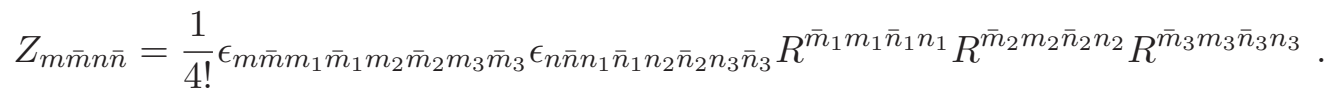

This tensor satisfies the identities

$$
Z_{m \bar{m} n \bar{n}}=Z_{n \bar{m} m \bar{n}}=Z_{m \bar{n} n \bar{m}}, \quad \nabla^{m} Z_{m \bar{m} n \bar{n}}=\nabla^{\bar{m}} Z_{m \bar{m} n \bar{n}}=0
$$

and is related to the third and fourth Chern-form $c_{3}, c_{4}$ as

$$
\begin{aligned}
Z_{m \bar{m}} & =i 2 Z_{m \bar{m} n}{ }^{n}=\frac{1}{2}\left(* c_{3}\right)_{m \bar{m}}, & Z & =i 2 Z_{m}{ }^{m}=*\left(J \wedge c_{3}\right), \\
*\left(c_{3} \wedge \omega_{i}\right) & =-2 Z_{m \bar{n} \omega_{i}{ }^{\bar{n} m}}, & Z_{m \bar{m} n \bar{n}} R^{\bar{m} m \bar{n} n} & =* c_{4},
\end{aligned}
$$

with $Z$ already given in (2.3). 
The tensor $Z_{m \bar{n} r \bar{s}}$ is the basic building block to display the corrected metric $G_{i j}^{T}$, the coupling $K_{i}^{T}$, and the volume $\mathcal{V}_{T}$. Explicitly they take the form

$$
\begin{aligned}
G_{i j}^{T}= & G_{i j}^{W}+256 \alpha^{2} \frac{1}{\mathcal{V}_{0}^{2}} \int_{Y_{4}} Z * 1 \int_{Y_{4}} \omega_{i m \bar{n}} \omega_{j}^{\bar{n} m} * 1 \\
& -256 \alpha^{2} \frac{1}{\mathcal{V}_{0}} \int_{Y_{4}}\left[Z \omega_{i m \bar{n}} \omega_{j}^{\bar{n} m}+12 Z_{m \bar{n} r \bar{s}} \omega_{j}^{\bar{n} m} \omega_{i}^{\bar{s} r}\right] * 1, \\
K_{i}^{T}= & K_{i}^{0}+\alpha^{2} \int_{Y_{4}}\left[\frac{K_{i}^{0}}{\mathcal{V}_{0}}(3 W-128 Z) * 1-1536 Z_{m \bar{n}} \omega_{i}^{\bar{n} m} * 1\right], \\
\mathcal{V}_{T}= & \mathcal{V}_{W}+256 \alpha^{2} \int_{Y_{4}} Z * 1,
\end{aligned}
$$

where all quantities are evaluated in the background $v_{0}^{i}$. The coefficients in the expressions for $G_{i j}^{T}, K_{i}^{T}$, and $\mathcal{V}_{T}$ first appear to be unrelated. However, they are in fact precisely taking values so as to ensure the identity

$$
\left(G_{i j}^{T}+\mathcal{V}_{T}^{-2} K_{i}^{T} K_{j}^{T}\right)=G_{a b}^{T}\left(\delta_{i}^{a}-\frac{1}{\mathcal{V}_{0}} v_{0}^{a} K_{i}^{0}\right)\left(\delta_{j}^{b}-\frac{1}{\mathcal{V}_{0}} v_{0}^{b} K_{j}^{0}\right),
$$

which holds in the background $v_{0}^{i}$. As we will demonstrate in the next section, this identity is one of the crucial ingredients to ensure supersymmetry of the three-dimensional effective action.

\subsection{Warp-factor scaling symmetry and integration}

In this subsection we will have a closer look at the couplings in (2.24). In particular, it was observed in [7] that the three-dimensional effective action permits a scaling symmetry involving the rescaling of the warp-factor. More precisely, the action turns out to be invariant under the symmetry

$$
W \rightarrow W+\Lambda, \quad v^{i} \rightarrow e^{-\alpha^{2} \Lambda} v^{i}
$$

for any scalar function $\Lambda=\Lambda\left(v^{i}\right)$ that can be space-time dependent. The scalars $v^{i}$ in (2.24) therefore have to appear with a covariant derivative (2.29), which can be extended to include the fluctuations by writing

$$
D v^{i}=d v^{i}+\alpha^{2} \mathcal{W}_{j} d v^{j} v^{i}, \quad \mathcal{W}_{j}=\frac{1}{\mathcal{V}} \int_{Y_{4}} \partial_{j} W * 1
$$

It is conceivable that this scaling invariance persists beyond the $\alpha$-order testable in the current reduction. It is also interesting to note that one can introduce a potential $\mathcal{W}$ for the connection in $(2.36)$ as

$$
\mathcal{W}_{j}=\partial_{j}\left(\frac{\mathcal{W}}{\mathcal{V}}\right), \quad \mathcal{W}\left(v^{i}\right)=\frac{1}{4 !} \int_{Y_{4}} W J^{4}
$$

where $J=v^{i} \omega_{i}$ contains the fluctuated Kähler moduli. 
The scaling symmetry fixes a number of the warp-factor dependent terms in (2.24) and one readily infers a potential $\mathcal{W}$ that appears in these couplings. However, there is one contribution proportional to $\int_{Y_{4}} W \omega_{i} \wedge \omega_{j} \wedge J \wedge J$ that appears to be special. It arises by expanding

$$
G_{i j}^{W}=-\frac{1}{2 \mathcal{V}} K_{i j}+\frac{1}{2 \mathcal{V}^{2}} K_{i} K_{j}-\frac{3}{4 \mathcal{V}} \int_{Y_{4}} W \omega_{i} \wedge \omega_{j} \wedge J \wedge J+\frac{3}{2 \mathcal{V}^{2}} K_{i j} \int W * 1,
$$

where we have used ${ }^{3}$

$$
* \omega_{i}=-\frac{1}{2} \omega_{i} \wedge J \wedge J+\frac{1}{6 \mathcal{V}} K_{i} J^{3} .
$$

At first, one might have suspected that all terms in (2.38) arise as derivatives of $\mathcal{W}$ as well. However, evaluating ${ }^{4}$

$$
\partial_{j} \int W \omega_{i} \wedge J^{3}=3 ! K_{i} \mathcal{W}_{j}+3 ! \frac{\mathcal{W}}{\mathcal{V}} K_{i j}, \quad \partial_{j} \mathcal{W}_{i}=\frac{1}{4 ! \mathcal{V}} \int_{Y_{4}}\left(\partial_{i} \partial_{j} W\right) J^{4},
$$

one infers that there is no term proportional to $\int W \omega_{i} \wedge \omega_{j} \wedge J^{2}$. This is a first example of a situation where one can connect couplings with zero and one index, but new structures arise at the two-index level. We discuss similar issues arising in the higher-derivative sector next.

In order to integrate terms in the higher-derivative sector, one might want to start with the scalar function

$$
\mathcal{Z}\left(v^{i}\right)=\frac{1}{4 !} \int_{Y_{4}} Z J^{4}=\int_{Y_{4}} J \wedge c_{3}
$$

where we have used (2.32) and view $\mathcal{Z}$ as a function of the fluctuated moduli $v^{i}$. It is then straightforward to derive

$$
\mathcal{Z}_{i}=\partial_{i} \mathcal{Z}=\int_{Y_{4}} \omega_{i} \wedge c_{3}=-2 \int_{Y_{4}} Z_{m \bar{n}} \omega_{i}^{\bar{n} m} * 1
$$

where we again inserted (2.32). Note that when written with the Chern-form $c_{3}$ it is obvious that $\mathcal{Z}_{i}$ is actually constant such that $\partial_{j} \mathcal{Z}_{i}=0$. Thus, in complete analogy to the warping terms, there appears to be no obvious potential that admits the two-index terms

$$
\int_{Y_{4}} Z_{m \bar{n} r \bar{s}} \omega_{j}^{\bar{n} m} \omega_{i}^{\bar{s} r} * 1, \quad \int_{Y_{4}} Z \omega_{i} \wedge \omega_{j} \wedge J \wedge J
$$

as derivatives. We will have to address precisely these obstacles when showing the supersymmetry of the effective action in next section.

To close this section let us point out that the two terms in (2.43) are just part of a set of higher derivative terms of the form

$$
\mathcal{X}_{i j k l}^{(r)}=\int_{Y_{4}} \omega_{i} \wedge \mathcal{R}_{m_{1} \bar{m}_{1}} \wedge \mathcal{R}_{m_{2} \bar{m}_{2}} \wedge \mathcal{R}_{m_{2} \bar{m}_{2}} \omega_{i}^{\bar{n}_{1} n_{1}} \omega_{k}^{\bar{n}_{2} n_{2}} \omega_{l}^{\bar{n}_{3} n_{3}}\left(\mathcal{Y}_{(r)}\right)_{n_{1} \bar{n}_{1} n_{2} \bar{n}_{2} n_{3} \bar{n}_{3}}^{m_{1} \bar{m}_{2} m_{2} \bar{m}_{2} m_{3} \bar{m}_{3}}
$$

\footnotetext{
${ }^{3}$ Note that this relation only holds for harmonic forms $\omega_{i}$.

${ }^{4} \mathrm{~A}$ simple way to show the first identity is to split the integral $\int W \omega_{i} \wedge J^{3} \propto \mathcal{W} K_{i}$, by using that $\omega_{i} \wedge J^{3}$ is harmonic.
} 
where the $\mathcal{Y}_{(r)}$ are defined to encode all possible index contractions of $m_{p}$ with $n_{q}$. The two terms in (2.43) arise when contracting a particular set of $\mathcal{X}_{i j k l}^{(r)}$ with $v^{k}$ and $v^{l}$. It would be very interesting to study the properties of such $\mathcal{X}_{i j k l}^{(r)}$. In particular, the variation of these terms with the moduli $v^{i}$ might uncover interesting relations. Furthermore, it is worth stressing that the terms $\mathcal{X}_{i j k l}^{(r)}$ including the contractions (2.43) depend on the chosen forms $\omega_{i}$, i.e. not just on the class of $\omega_{i}$, for all appearing two-forms. In our study the $\omega_{i}$ were always harmonic, but it would be interesting to check if there are linear combinations of the $\mathcal{X}_{i j k l}^{(r)}$ or its $v^{p}$ contractions that only depend on the cohomology class of the two-forms.

\section{Demonstrating the supersymmetric structure}

In this section we determine the Kähler potential and complex coordinates compatible with $\mathcal{N}=2$ supersymmetry in three dimensions. Our starting point will be the threedimensional effective action (2.24) obtained by dimensional reduction. We discuss its supersymmetric structure both in the frame when working with vectors $A^{i}$ and in the dual frame when the vectors are replaced by scalars $\rho_{i}$.

\subsection{Comparing the reduction result with $\mathcal{N}=2$ supergravity}

It turns out to be convenient to first work with three-dimensional vector multiplets with bosonic fields $\left(L^{i}, A^{i}\right)$ and only later switch to chiral multiplets with complex scalars $T_{i}$. The kinetic terms of an ungauged $\mathcal{N}=2$ supergravity theory can be written as

$$
S_{\text {kin }}=\int_{\mathcal{M}_{3}}\left(\frac{1}{2} R * 1+\frac{1}{4} \tilde{K}_{L^{i} L^{j}} d L^{i} \wedge * d L^{j}+\frac{1}{4} \tilde{K}_{L^{i} L^{j}} F^{i} \wedge * F^{j}\right)
$$

In this expression $\tilde{K}_{L^{i} L^{j}}$ can be determined from a so-called kinetic potential $\tilde{K}(L)$ via $\tilde{K}_{L^{i} L^{j}}=\partial_{L^{i}} \partial_{L^{j}} \tilde{K}$. Dualising the vector $A^{i}$ in the vector multiplet one can translate the three-dimensional theory into an action for complex scalars $T_{i}$ with kinetic terms given by a Kähler potential $K(T, \bar{T})$. The action then takes the form

$$
S_{\mathrm{kin}}=\int_{\mathcal{M}_{3}}\left(\frac{1}{2} R * 1-K_{T_{i} \bar{T}_{j}} d T_{i} \wedge * d \bar{T}_{j}\right),
$$

where $K_{T_{i} \bar{T}_{j}}=\partial_{T_{i}} \partial_{\bar{T}_{j}} K$ is the Kähler metric. Note that $\operatorname{Re} T_{i}, K$ and $L^{i}, \tilde{K}$ are related by a Legendre transform as

$$
T_{i}=\tilde{K}_{L^{i}}+i \rho_{i}, \quad K=\tilde{K}-\frac{1}{2}\left(T_{i}+\bar{T}_{i}\right) L^{i}
$$

where $\rho_{i}$ is the three-dimensional scalar dual to the vector $A^{i}$. One can now straightforwardly derive that $K_{T_{i} \bar{T}_{j}}=-\frac{1}{4} \tilde{K}^{L^{i} L^{j}}$, which uses the inverse of $\tilde{K}_{L^{i} L^{j}}$. Note that $K$ is independent of the scalar $\rho_{i}$ and thus a function $K\left(\operatorname{Re} T_{i}\right)$. It is useful to recall the inverse transformation

$$
L^{i}=-2 K_{T_{i}}
$$


where $K_{T_{i}}=\partial_{T_{i}} K$. The theory formulated in the $T_{i}$ coordinates can admit a scalar potential of the form

$$
S_{\text {pot }}=-\int_{\mathcal{M}_{3}}\left(K^{T_{i} \bar{T}_{j}} \partial_{T_{i}} \mathcal{T} \partial_{\bar{T}_{j}} \mathcal{T}-\mathcal{T}^{2}\right) * 1+e^{K}\left(K^{T_{i} \bar{T}_{j}} D_{T_{i}} W \overline{D_{T_{j}} W}-4\left|W^{2}\right|\right) * 1,
$$

where $K^{T_{i} \bar{T}_{j}}$ is the inverse of the Kähler metric $K_{T_{i} \bar{T}_{j}}$. Here $\mathcal{T}$ is a real function of the fields $T_{i}$, while $W$ is a holomorphic superpotential in the $T_{i}$. The latter transforms non-trivially under Kähler transformations and therefore appears with the Kähler covariant derivative $D_{T_{i}} W=\partial_{T_{i}} W+K_{T_{i}} W$.

To read off $\tilde{K}_{L^{i} L^{j}}$ we compare the action (3.1) with the result from the dimensional reduction (2.24). We first read off the coefficient of the $F^{i} \wedge * F^{j}$ term and identify

$$
\tilde{K}_{L^{i} L^{j}} \mid=-\frac{1}{2} \mathcal{V}_{T}^{2} G_{i j}^{T}
$$

Here we have used the notation $f\left(v^{i}\right) \mid=f\left(v_{0}^{i}\right)$, i.e. the vertical dash denotes evaluation in the background setting all fluctuations $\delta v^{i}=0$. Supersymmetry implies that for the correct definition of $L^{i}$, this metric has to match the one in front of $d L^{i} \wedge * d L^{j}$. Applied to $(2.24)$ this implies the relation

$$
\mathcal{V}_{T}^{2} G_{i j}^{T}=\left(G_{c d}^{T}+\mathcal{V}_{T}^{-2} K_{c}^{T} K_{d}^{T}\right)\left(\delta_{a}^{c}+v_{0}^{c} \mathcal{W}_{a}^{0}\right)\left(\delta_{c}^{d}+v_{0}^{d} \mathcal{W}_{b}^{0}\right) \frac{\partial v^{a}}{\partial L^{i}}\left|\frac{\partial v^{b}}{\partial L^{j}}\right|
$$

where $\mathcal{W}_{a}^{0}=\mathcal{W}_{a} \mid$ is defined in (2.36) and evaluating in the background. Then using (2.34) we find that

$$
\partial_{j} L^{i}\left|\equiv \frac{\partial L^{i}}{\partial v^{j}}\right|=\frac{1}{\mathcal{V}_{T}}\left(\delta_{k}^{i}-\frac{v_{0}^{i}}{\mathcal{V}_{0}} K_{k}^{0}\right)\left(\delta_{j}^{k}+v_{0}^{k} \mathcal{W}_{a}^{0}\right)
$$

where as above we abbreviate derivatives with respect to $v^{i}$ as $\partial_{i} \equiv \frac{\partial}{\partial v^{i}}$. It turns out to be complicated to integrate this condition. This can be traced back to the fact that there is an evaluation and, as we discuss below, the fundamental objects to define $L^{i}$ itself might be more involved. Nevertheless, we can already make some interesting observations. Firstly, the higher-curvature corrections only appear through $\mathcal{V}_{T}$ in (3.8). One suspects that this can only be true in the background. In fact, we might imagine that $\partial_{j} L^{i}$ contains a term

$$
\partial_{j} L^{i} \supset v^{i} \int_{Y_{4}}\left[Z_{m \bar{n}} \omega_{j}^{\bar{n} m}-2 Z_{m \bar{n} r \bar{s}} \omega_{j}^{\bar{n} m} \omega_{k}^{\bar{s} r} v^{k}\right] *^{(0)} 1
$$

which trivially gives zero when evaluated at $v_{0}^{i}$. Terms of this type, however, will turn out to be crucial in order to determine the underlying objects of the theory. In contrast, artificially switching off the higher-curvature corrections in (3.8) one finds that the $L^{i}$ in the presence of warping actually takes the simple form

$$
L^{i}=\frac{v^{i}}{\mathcal{V}_{W}}
$$

where $\mathcal{V}_{W}$ is the warped volume (2.28) now evaluated as a function of the perturbed $v^{i}$. 
As a second requirement of supersymmetry we note that (3.3) implies

$$
\partial_{i} \operatorname{Re} T_{j}\left|=\tilde{K}_{L^{j} L^{k}} \partial_{i} L^{k}\right|
$$

Using (3.6) and (3.8) we conclude that

$$
\begin{aligned}
\partial_{j} \operatorname{Re} T_{i} \mid= & K_{i j}^{0}+3 \alpha^{2} K_{i}^{0} \mathcal{W}_{j}^{0}+\frac{3}{2} \alpha^{2} \int_{Y_{4}} W \omega_{i} \wedge \omega_{j} \wedge J \wedge J \mid \\
& -256 \alpha^{2} \frac{1}{\mathcal{V}_{0}} K_{i j}^{0} \mathcal{Z}_{0}-1536 \alpha^{2} \frac{1}{\mathcal{V}_{0}} K_{j}^{0} \mathcal{Z}_{i}^{0} \\
& +256 \alpha^{2} \int_{Y_{4}} Z \omega_{i} \wedge \omega_{j} \wedge J \wedge J\left|+6144 \alpha^{2} \int_{Y_{4}} \omega_{i}{ }^{\bar{n} m} \omega_{j}{ }^{\bar{r} r} Z_{m \bar{n} r \bar{s}} * 1\right|
\end{aligned}
$$

where $K_{i j}^{0}$ and $K_{i}^{0}$ are introduced in $(2.27)$ and evaluated at $v_{0}^{i}$.

\subsection{Kähler potential and coordinates as a $\delta v$ expansion}

In the previous section we have deduced the expressions for $\partial L^{i} / \partial v^{j}$ and $\partial \operatorname{Re} T_{j} / \partial v^{i}$ when evaluated in the background $v^{i}=v_{0}^{i}$. We will next try to infer directly the coordinates $T_{i}$ and the Kähler potential $K$. In order to do this we view $T_{i}$ and $K$ as being given by an expansion both in $\alpha$ and $\delta v^{i}$ by writing

$$
\begin{aligned}
\operatorname{Re} T_{i} & =\operatorname{Re} T_{i}^{(0)}+\alpha^{2} \operatorname{Re} T_{i}^{(2)}, & \operatorname{Re} T_{i}^{(2)} & =\operatorname{Re} T_{i}^{(2)}\left|+\partial_{j} \operatorname{Re} T_{i}^{(2)}\right| \delta v^{j}+\frac{1}{2} \partial_{j} \partial_{k} \operatorname{Re} T_{i}^{(2)} \mid \delta v^{j} \delta v^{k}, \\
K & =K^{(0)}+\alpha^{2} K^{(2)}, & K^{(2)} & =K^{(2)}\left|+\partial_{j} K^{(2)}\right| \delta v^{j}+\frac{1}{2} \partial_{j} \partial_{k} K^{(2)} \mid \delta v^{j} \delta v^{k} .
\end{aligned}
$$

In the following we derive as much information as possible about the coupling functions that appear in this expansion by comparing to the reduction result.

As a first step, recall that the zeroth order result in $\alpha$ was already determined in $[18,19]$. With our above expressions one can check that

$$
K^{(0)}=-3 \log (\mathcal{V}), \quad \operatorname{Re} T_{i}^{(0)}=K_{i},
$$

where now $\mathcal{V}$ and $K_{i}$ depend on the varying $v^{i}$. At the next order in $\alpha$ we note that there are only few objects with zero or one index $i$ that are non-trivial in the background. More precisely, one can write

$$
K^{(2)} \mid=\frac{\mu_{1}}{\mathcal{V}_{0}} \mathcal{Z}_{0}+\frac{\mu_{2}}{\mathcal{V}_{0}} \mathcal{W}_{0}
$$

where $\mathcal{Z}$ and $\mathcal{W}$ are defined in (2.41) and (2.37). The constants $\mu_{1}, \mu_{2}$ are undetermined at this point. Clearly, the constant shifts in $K$ are unimportant for the derivation of the Kähler metric. However, the form of (3.15) might hint towards the fully moduli-dependent form of $K$. To fix the coefficients $\mu_{2}$ one might be inclined to use the scaling symmetry (2.35). Together with the classical form of $K$ one then infers that an invariant $K$ requires $\mu_{2}=-12$. 
We can proceed similarly for the one-index quantities. We first make an ansatz using all one-index building blocks we have encountered so far by setting

$$
\begin{gathered}
\operatorname{Re} T_{i}^{(2)} \mid=\tilde{\nu}_{1} \mathcal{Z}_{i}+\tilde{\nu}_{2} \mathcal{V}_{0} \mathcal{W}_{i}^{0}+\tilde{\nu}_{3} K_{i}^{0} \mathcal{Z}_{0}+\tilde{\nu}_{4} K_{i}^{0} \mathcal{W}_{0}, \\
\partial_{i} K^{(2)} \mid=\frac{\tilde{\mu}_{1}}{\mathcal{V}_{0}} \mathcal{Z}_{i}+\tilde{\mu}_{2} \mathcal{W}_{i}^{0}+\frac{\tilde{\mu}_{3}}{\mathcal{V}_{0}} K_{i}^{0} \mathcal{Z}_{0}+\frac{\tilde{\mu}_{4}}{\mathcal{V}_{0}} K_{i}^{0} \mathcal{W}_{0} .
\end{gathered}
$$

The constant coefficients $\tilde{\nu}_{\alpha}, \tilde{\mu}_{\alpha}$ are not determined at this point, since there are no direct relations fixing the background values of $T_{i}$ and $\partial_{i} K$. To fix at least some of the coefficients in (3.16) one can again use the symmetry (2.35). Note that $T_{i}$ are proper complex coordinates that should be invariant under (2.35). This suggests that $\tilde{\nu}_{4}=3$ and $\tilde{\nu}_{2}=0$, where we have used that the leading contribution to $T_{i}$ is of third power in $v^{i}$ as in (3.14). In contrast, we note that $K$ should be invariant under (2.35), while $\partial_{i} K^{(2)}$ should transform as a derivative and therefore contain the connection $\mathcal{W}_{i}$. Using again the leading form (3.14) and the expression (2.37) one concludes $\tilde{\mu}_{2}=-12$ and $\tilde{\mu}_{4}=0$.

In contrast to (3.15) and (3.16) the form of $\partial_{j} \operatorname{Re} T_{i}^{(0)} \mid$ and $\partial_{j} \operatorname{Re} T_{i}^{(2)} \mid$ are fully fixed by the reduction and are trivially read off from (3.13) with

$$
\begin{aligned}
\partial_{j} \operatorname{Re} T_{i}^{(2)} \mid= & 3 K_{i}^{0} \mathcal{W}_{j}^{0}+\frac{3}{2} \int_{Y_{4}} W \omega_{i} \wedge \omega_{j} \wedge J \wedge J \mid-256 \frac{1}{\mathcal{V}_{0}} K_{i j}^{0} \mathcal{Z}_{0}-1536 \frac{1}{\mathcal{V}_{0}} K_{j}^{0} \mathcal{Z}_{i}^{0} \\
& +256 \int_{Y_{4}} Z \omega_{i} \wedge \omega_{j} \wedge J \wedge J\left|+6144 \int_{Y_{4}} \omega_{i}^{\bar{n} m} \omega_{j}^{\bar{s} r} Z_{m \bar{n} r \bar{s}} * 1\right|
\end{aligned}
$$

All other remaining terms in the expansion (3.16) are also not fully determined by our results obtained from the reduction. However, we can use (3.4) to show that the general relation

$$
L^{i}=-2 \frac{\partial K}{\partial T_{i}}=-\frac{\partial K}{\partial v^{j}} \frac{\partial v^{j}}{\partial \operatorname{Re} T_{i}}
$$

together with (3.16) gives

$$
\begin{aligned}
L^{(2) i}= & -K^{i j} \partial_{j} K^{(2)}\left|-\frac{1}{\mathcal{V}} v^{j} K^{i k} \partial_{k} T_{j}^{(2)}\right|+K_{j l m} K^{i l} K^{k m} \partial_{k} K^{(2)} \mid \delta v^{j} \\
& -K^{i k} \partial_{j} \partial_{k} K^{(2)}\left|\delta v^{j}-\frac{1}{\mathcal{V}} K^{i k} \partial_{k} T_{j}^{(2)}\right| \delta v^{j}+\frac{1}{\mathcal{V}^{2}} K_{j} v^{l} K^{i k} \partial_{k} T_{l}^{(2)} \mid \delta v^{j} \\
& +\frac{1}{\mathcal{V}} K_{j m n} K^{i m} K^{l n} v^{k} \partial_{l} T_{k}^{(2)}\left|\delta v^{j}-\frac{1}{\mathcal{V}} K^{i l} v^{k} \partial_{j} \partial_{l} T_{k}^{(2)}\right| \delta v^{j}+\mathcal{O}\left(\delta v^{2}\right) .
\end{aligned}
$$

From this it is straightforward to evaluate $\partial_{i} L^{j}$ and compare the result with (3.8) in the background $v^{i}=v_{0}^{i}$. One then infers that the coefficients in (3.16) have to satisfy the relation

$$
\begin{aligned}
\partial_{i} \partial_{j} \operatorname{Re} & T_{k}^{(2)} v^{k}\left|-\mathcal{V} K_{i j k} K^{k l} \partial_{l} K^{(2)}\right|+\mathcal{V} \partial_{j} \partial_{k} K^{(2)} \mid \\
= & 9 \frac{1}{\mathcal{V}_{0}} K_{i j}^{0} \mathcal{W}_{0}+18 \mathcal{V}_{0} \mathcal{W}_{(i}^{0} K_{j)}^{0}+12 \mathcal{V}_{0} K_{i j k}^{0} K_{0}^{k l} \mathcal{W}_{l}^{0}-\frac{3}{2} \int W \omega_{i} \wedge \omega_{j} \wedge J \wedge J \mid \\
& -256 \int Z \omega_{i} \wedge \omega_{j} \wedge J \wedge J \mid+3072 \frac{1}{\mathcal{V}_{0}} K_{(i}^{0} \mathcal{Z}_{i)}^{0}-1536 \frac{1}{\mathcal{V}_{0}^{2}} K_{i}^{0} K_{j}^{0} \mathcal{Z}_{0} \\
& -6144 \int \omega_{i}^{\bar{n} m} \omega_{j}^{\bar{s} r} Z_{m \bar{n} r \bar{s}} * 1 \mid+1536 K_{i j k}^{0} K_{0}^{k l} \mathcal{Z}_{l}^{0} .
\end{aligned}
$$


Imposing these conditions then implies that we match the metric (3.6). Note that this analysis can be carried out independent of any gauge fixing of the scaling symmetry (2.35). Also note that our first-order analysis does neither uniquely fix the Kähler coordinates nor the Kähler metric. This can be traced back to the fact that we performed the dimensional reduction only to leading order in the fluctuations $\delta v^{i}$.

In order to fix the coefficients in (3.16) further, one can try to impose conditions that might hold also at the higher-derivative level. For example, one may suspect that a noscale condition holds even when including $\alpha$-corrections to the action. In three space-time dimensions such a condition reads

$$
K_{T_{i}} K^{T_{i} \bar{T}_{j}} K_{\bar{T}_{j}}=4
$$

It ensures that in the scalar potential (3.5) the negative $-4|W|^{2}$ term cancels for a superpotential independent of $T_{i}$. Using (3.4) and $K^{T_{i} \bar{T}_{j}}=-4 \tilde{K}_{L^{i} L^{j}}$ one rewrites (3.21) as

$$
L^{i} \tilde{K}_{L^{i} L^{j}} L^{j}=-4 .
$$

In the background this expression can be evaluated by using (3.19) together with (3.17) to yield the condition ${ }^{5}$

$$
\partial_{i} K^{(2)} v^{i} \mid=2304 \frac{1}{\mathcal{V}_{0}} \mathcal{Z}_{0}-12 \mathcal{W}_{i}^{0} v_{0}^{i}
$$

Keeping in mind that we have few objects with zero or one index, one can use this condition as a further motivation to make an ansatz for the Kähler potential and match the coefficients. This will be considered in the following section.

\subsection{Completing the Kähler potential and complex coordinates}

In this final subsection we comment on the completion of the Kähler potential and complex coordinates as a closed expression in Kähler deformations. Our goal is to replace the $\delta v^{i}$ expansion (3.16) with an appropriate ansatz hinting towards the underlying structure of the higher-derivative reduction. It should be stressed that we are only able to fully justify the leading terms. However, we will also discover an intriguing interplay between warping effects and higher-curvature terms.

To begin with, let us propose an ansatz for the Kähler potential. We have noted in (3.15) that there are only few objects without indices. Using the quantities introduced in (2.37) and (2.41) we suggest

$$
\begin{aligned}
K & =-3 \log \left(\int_{Y_{4}} e^{4 \alpha^{2} W} * 1+256 \mu \alpha^{2} \int_{Y_{4}} Z * 1\right) \\
& =-3 \log \left(\mathcal{V}+256 \mu \alpha^{2} \mathcal{Z}+4 \alpha^{2} \mathcal{W}+\mathcal{O}\left(\alpha^{4}\right)\right)
\end{aligned}
$$

\footnotetext{
${ }^{5}$ We note also that a similar set constraints $\operatorname{Re} T_{i} \operatorname{Re} T_{j} G^{i j}\left|=L^{i} L^{j} G_{i j}^{-1}\right|=L^{i} \operatorname{Re} T_{i} \mid=4$ and $\partial_{k}\left(L^{i} \operatorname{Re} T_{i}\right) \mid=0$ can all be satisfied if we demand (3.24) as well as

$$
\operatorname{Re} T_{i}^{(2)}\left|=\partial_{i} K^{(2)}\right|-\frac{1}{3} K_{i} \partial_{j} K^{(2)} v^{j} \mid+12 \mathcal{W}_{i}^{0}+3 K_{i}^{0} \mathcal{W}_{0}-4 \frac{1}{\mathcal{V}_{0}} K_{i}^{0} \mathcal{W}_{j}^{0} v_{0}^{j}+256 \frac{1}{\mathcal{V}} K_{i}^{0} \mathcal{Z}_{0}
$$


where the functions that appear are now viewed as being dependent on the fields $v^{i}$. In this expression we fixed the factor in front of $\mathcal{W}$ by the fact that $K$ has to be invariant under the symmetry (2.35). The factor in front of the $\mathcal{Z}$ term is not fixed a priori and we have introduced the constant $\mu$ to capture this freedom. Let us stress that it is straightforward to compute the $v^{i}$ derivatives of $K$ as defined in (3.25). In particular, one finds

$$
\partial_{i} K=-3 \frac{1}{\mathcal{V}} K_{i}+768 \mu \alpha^{2} \frac{1}{\mathcal{V}} \mathcal{Z} K_{i}-768 \mu \alpha^{2} \frac{1}{\mathcal{V}} \mathcal{Z}_{i}-12 \alpha^{2} \mathcal{W}_{i}
$$

Clearly, in order to compute the actual Kähler metric we also have to supplement an ansatz for the complex coordinates $T_{i}$. The involved form of the Kähler metric determined from the dimensional reduction (2.24) and the rather simple form of the Kähler potential (3.25) as a function of the $v^{i}$ suggests that the $T_{i}$ have to capture most of the non-trivial information about the $\mathcal{N}=2$ system.

To get some intuitive information about $T_{i}$, we note that these coordinates are expected to linearise the action of M5-brane instantons on divisors $D_{i}$. In fact, as discussed in [39] a holomorphic superpotential of the schematic form $W \propto e^{-T_{i}}$ can be induced by such instanton effects. This implies that the $T_{i}$ are expected to be integrals over divisors $D_{i}$. We therefore suggest that they take the form

$$
T_{i}=\int_{D_{i}}\left(\frac{1}{3 !} e^{3 \alpha^{2} W} J \wedge J \wedge J+1536 \alpha^{2} F_{6}\right)+i \rho_{i},
$$

where $D_{i}$ are $h^{1,1}\left(Y_{4}\right)$ divisors of $Y_{4}$ that span the homology $H_{2}\left(Y_{4}, \mathbb{R}\right)$. The six-form $F_{6}$ in this expression is a function of degrees of freedom associated with the internal space metric and will be responsible for the more complicated higher derivative structures (2.43). It is constrained by a relation to the fourth Chern form $c_{4}$ such that $F_{6}$ determines the non harmonic part of $c_{4}$ as

$$
c_{4}=H c_{4}+i \partial \bar{\partial} F_{6}
$$

This is in analogy to the quantity $F_{4}$ introduced for $c_{3}$ in (2.7). Note that (3.28) leaves the harmonic and exact part of $F_{6}$ unfixed and we will discuss constraints on these pieces in more detail below. The justification of the first term in $\operatorname{Re} T_{i}$ is simpler. It captures the warped volume of an M5-brane wrapped on $D_{i}$. In fact, the power of the warp-factor turns out to be appropriate to ensure invariance under the scaling symmetry (2.35), in accord with the expectation that $T_{i}$ is invariant under this symmetry. Remarkably, this definition of the Kähler coordinates as $D_{i}$ integrals will help us to obtain the couplings $\int e^{3 \alpha^{2} W} J \wedge J \wedge \omega_{i} \wedge \omega_{j}$, which, as we stressed in subsection 2.5, cannot be obtained as $v^{i}$ derivatives of the considered $Y_{4}$-integrals. Note that the following discussion of the warping is inspired by [33]. Here we will adapt and extend the arguments of [33] and include the higher-curvature pieces. Interestingly they turn out to complete the analysis in an elegant and non-trivial fashion.

In order to evaluate the derivatives of $T_{i}$ with respect to $v^{i}$ and to make contact with the Kähler metric found in (2.24), we have to rewrite the integrals over $D_{i}$ into integrals over $Y_{4}$. Due to the appearance of the warp-factor and the non-closed form $F_{6}$ in (3.27) this is not straightforward. In particular, one cannot simply use Poincaré duality and write 
$T_{i}$ as an integral over $Y_{4}$ with inserted $\omega_{i}$. Of course, it is always possible to write $T_{i}$ as a $Y_{4}$ integral when inserting a delta-current localised on $D_{i}$, i.e.

$$
\operatorname{Re} T_{i}=\int_{Y_{4}}\left(\frac{1}{3 !} e^{3 \alpha^{2} W} J \wedge J \wedge J+1536 \alpha^{2} F_{6}\right) \wedge \delta_{i},
$$

where $\delta_{i}$ is the $(1,1)$-form delta-current that restricts to the divisor $D_{i}$. Appropriately extending the notion of cohomology to include currents $[40,41]$, we can now ask how much $\delta_{i}$ differs from the harmonic form $\omega_{i}$ in the same class. In fact, any current $\delta_{i}$ is related to the harmonic element of the same class $\omega_{i}$ by a doubly exact piece as

$$
\delta_{i}=\omega_{i}+i \partial \bar{\partial} \lambda_{i} .
$$

This equation should be viewed as relating currents. Importantly, as we assume $D_{i}$ and hence $\delta_{i}$ to be $v^{i}$-independent, the $v^{i}$ dependence of the harmonic form $\omega_{i}$ and the current $\lambda_{i}$ has to cancel such that $\partial_{j} \omega_{i}=-i \partial \bar{\partial} \partial_{j} \lambda_{i}$. Importantly, once we determine $\partial_{j} \operatorname{Re} T_{j}$ we can express the result as $Y_{4}$-integrals without invoking currents. We therefore need to understand how each part of $T_{i}$ varies under a change of moduli. This will also fix the numerical factor in front of $F_{6}$ in (3.27).

In order to take derivatives of $T_{i}$ we first use the fact that $D_{i}$ and hence $\delta_{i}$ are independent of the moduli $v^{i}$, which implies

$$
\partial_{j} \operatorname{Re} T_{i}=\int_{Y_{4}}\left(\frac{1}{2} e^{3 \alpha^{2} W} \omega_{j} \wedge J \wedge J+\frac{1}{2} \alpha^{2} \partial_{j} W J \wedge J \wedge J+1536 \alpha^{2} \partial_{j} F_{6}\right) \wedge \delta_{i} .
$$

We next claim that we can replace $\delta_{i}$ with $\omega_{i}$ such that finally

$\partial_{j} \operatorname{Re} T_{i}=\frac{1}{2} \int_{Y_{4}} e^{3 \alpha^{2} W} \omega_{i} \wedge \omega_{j} \wedge J \wedge J+\frac{1}{2} \alpha^{2} \int_{Y_{4}} \partial_{j} W \omega_{i} \wedge J \wedge J \wedge J+1536 \alpha^{2} \int_{Y_{4}} \omega_{i} \wedge \partial_{j} F_{6}$.

Note that by using (3.30) the two expressions (3.31) and (3.32) only differ by a term involving $\partial \bar{\partial} \lambda_{i}$. By partial integration this term is proportional to

$$
\begin{aligned}
\int_{Y_{4}} \lambda_{i} \partial \bar{\partial}\left(\frac{1}{2} e^{3 \alpha^{2} W} \omega_{j} \wedge J \wedge J+\frac{1}{2} \alpha^{2} \partial_{j} W J \wedge J \wedge J+1536 \alpha^{2} \partial_{j} F_{6}\right) \\
=\int_{Y_{4}} \lambda_{i}\left(\frac{1}{2} \partial \bar{\partial}\left(e^{3 \alpha^{2} W}\right) \omega_{j} \wedge J \wedge J+\frac{1}{2} \alpha^{2} \partial \bar{\partial}\left(\partial_{j} W\right) J \wedge J \wedge J+1536 \alpha^{2} \partial \bar{\partial} \partial_{j} F_{6}\right) .
\end{aligned}
$$

It is now straightforward to see that the terms multiplying $\lambda_{i}$ are simply the $\partial_{j}$ derivative of the warp-factor equation (2.9). One first writes (2.9) as

$$
d^{\dagger} d e^{3 \alpha^{2} W} *_{8} 1-\alpha^{2} Q_{8}=-\frac{1}{3} i \partial \bar{\partial}\left(e^{3 \alpha^{2} W}\right) \wedge J \wedge J \wedge J-\alpha^{2} Q_{8} .
$$

Then one takes the $v^{j}$-derivative of (3.34) by using the fact that $Q_{8}$ is given via $(2.10)$ and (3.28). The moduli dependence of $Q_{8}$ only arises from the term involving $F_{6}$, i.e. one has $\partial_{i} Q_{8}=i 3072 \partial \bar{\partial} \partial_{i} F_{6}$. Hence one finds exactly the terms in (3.33) such that this $\lambda_{i}$ dependent part of the $T_{i}$ variation vanishes due to the warp-factor equation (2.9). 
The final expression (3.32) is written using (2.27) and (2.37) as

$$
\partial_{j} \operatorname{Re} T_{i}=\frac{1}{2} \int_{Y_{4}} e^{3 \alpha^{2} W} \omega_{i} \wedge \omega_{j} \wedge J \wedge J+3 \alpha^{2} K_{i} \mathcal{W}_{j}+1536 \alpha^{2} \int_{Y_{4}} \omega_{i} \wedge \partial_{j} F_{6}
$$

The $L^{i}$ coordinates are then computed using (3.18) by inserting (3.26) and (3.35). This gives the result

$$
L^{i}=\frac{v^{i}}{\mathcal{V}}-\alpha^{2} \frac{v^{i}}{\mathcal{V}^{2}}(3 \mathcal{W}+256 \mu \mathcal{Z})+1536 \alpha^{2} \frac{K^{i j}}{\mathcal{V}}\left(\mathcal{Z}_{j}-\int_{Y_{4}} J \wedge \partial_{j} F_{6}\right) .
$$

It is then straightforward to derive

$$
\begin{aligned}
\partial_{j} L^{i}= & \frac{\delta_{j}^{i}}{\mathcal{V}}-\frac{v^{i} K_{j}}{\mathcal{V}^{2}}-\frac{\delta_{j}^{i}}{\mathcal{V}^{2}}(3 \mathcal{W}+256 \mu \mathcal{Z})-\frac{1}{\mathcal{V}} v^{i}\left(3 \mathcal{W}_{j}+256 \mu \mathcal{Z}_{j}\right)+\frac{1}{\mathcal{V}^{3}} K_{j} v^{i}(3 \mathcal{W}+512 \mu \mathcal{Z}) \\
& -\alpha^{2} \frac{1}{\mathcal{V}} 768 \mu K^{i m} K^{k n} K_{m n j} \mathcal{Z}_{k}-\alpha^{2} \frac{1}{\mathcal{V}_{0}^{2}} 768 \mu K^{i k} K_{j} \mathcal{Z}_{k} \\
& +\alpha^{2} \frac{1}{\mathcal{V}} 1536 K^{i m} K^{k n} K_{m n j} \int_{Y_{4}} J \wedge \partial_{k} F_{6}+\alpha^{2} \frac{1}{\mathcal{V}^{2}} 1536 K^{-1 i k} K_{j} \int_{Y_{4}} J \wedge \partial_{k} F_{6} \\
& -\alpha^{2} \frac{1}{\mathcal{V}} 1536 K^{-1 i k} \int_{Y_{4}} \omega_{j} \wedge \partial_{k} F_{6}-\alpha^{2} \frac{1}{\mathcal{V}} 1536 K^{-1 i k} \int_{Y_{4}} J \wedge \partial_{j} \partial_{k} F_{6} .
\end{aligned}
$$

This allows to determine the derivatives of $F_{6}$ by comparing (3.8) and (3.13) with (3.37) and (3.35). We find that

$$
\begin{aligned}
& \int_{Y_{4}} \omega_{i} \wedge \partial_{j} F_{6} \mid=4 \int_{Y_{4}} Z_{m \bar{n} r \bar{s}} \omega_{i}^{\bar{n} m} \omega_{j}^{\bar{s} r} * 1+\frac{1}{3 !} \int_{Y_{4}} Z \omega_{i} \wedge \omega_{j} \wedge J \wedge J-\frac{K_{i j}}{3 ! \mathcal{V}} \mathcal{Z}-\frac{1}{\mathcal{V}} K_{j} \mathcal{Z}_{i} \\
& \int_{Y_{4}} J \wedge \partial_{i} \partial_{j} F_{6} \mid=-4 \int_{Y_{4}} Z_{m \bar{n} r \bar{s}} \omega_{i}^{\bar{n} m} \omega_{j}^{\bar{s} r} * 1-\frac{1}{3 !} \int_{Y_{4}} Z \omega_{i} \wedge \omega_{j} \wedge J \wedge J \\
& -\mu \frac{1}{3 ! \mathcal{V}} K_{i j} \mathcal{Z}-(1-\mu) \frac{1}{\mathcal{V}^{2}} K_{i} K_{j} \mathcal{Z}+(2-\mu) \frac{1}{\mathcal{V}} K_{(i} \mathcal{Z}_{j)}+\frac{1}{2}(2-\mu) K_{i j k} K^{k l} \mathcal{Z}_{l},
\end{aligned}
$$

in order for the results to match. This implies that the Kähler potential (3.25) and coordinates (3.27) yield the metric matching with the reduction result.

The result (3.38) still depends on the free parameter $\mu$ introduced in the Kähler potential (3.25). Clearly, one expects that such a freedom is not fundamental, but rather due to the fact that we are only able to partially check the result. A dimensional reduction including fluctuations to higher order is likely fixing $\mu$ unambiguously. Alternatively, we can impose the no-scale condition (3.21), which we presume persists at higher curvature level. This implies that $\mu=1$.

Let us note that the definition contains two ambiguities. Firstly, we did not specify the divisor basis $D_{i}$ spanning $H_{2}\left(Y_{4}, \mathbb{R}\right)$. This can be shifted by a boundary of a seven-chain $\Gamma_{i}$ without changing the class as

$$
D_{i} \rightarrow D_{i}+\partial \Gamma_{i}
$$


This would result in a different choice for the currents $\delta_{i}$ and $\lambda_{i}$ in (3.30). The result is a modification of the $\mathcal{N}=2$ coordinates $T_{i}$ given in (3.29). However, as we have shown above, only the harmonic representative of the class enters in the variation $\partial_{j} T_{i}$, while $\lambda_{i}$ drops out due to the warp factor equation. In other words, the transformation (3.39) is actually a symmetry of the Kähler metric. Secondly, the constraint (3.28) is invariant under shifts of $F_{6}$ by six-forms $\eta_{6}$, which get annihilated by the derivatives. In other words, one might transform

$$
F_{6} \rightarrow F_{6}+\eta_{6}, \quad \bar{\partial} \eta_{6}=\partial \eta_{6}=0 .
$$

Clearly, this transformation will in general not respect (3.38). These conditions, however, constrain only the harmonic part of $F_{6}$ and allow for the the symmetry

$$
F_{6} \rightarrow F_{6}+d \tilde{\eta}_{4}
$$

It would be interesting to investigate the implication of the symmetries (3.39) and (3.41) in greater detail. This is particularly interesting when including a superpotential explicitly depending on the coordinates $T_{i}$.

The presence of the $F_{6}$ term in (3.27) implies, by the above relationship between $T_{i}$ and the action of a probe M5-brane on $D_{i}$, that higher-derivative corrections are relevant in the M5-brane action. Corrections of this type are also required for gravitational anomaly cancellation [42-44] for an M5-brane in the background of eleven-dimensional supergravity. From this anomaly analysis additional metric dependent contributions to the M5-brane action that are related to certain topological classes are expected, in a way similar to the relationship between $F_{6}$ and $c_{4}$. In future work it would be interesting to see if this analysis can be used to infer a more direct definition of the $F_{6}$ part of the correction in (3.27) and so prove the constraints (3.38) that are necessary in our analysis.

\section{Conclusions}

In this work we continued the study of the three-dimensional effective action obtained form dimensionally reducing M-theory on eight-dimensional compact manifolds initiated in $[4,7]$. The background solutions contain a warped product of an internal manifold $Y_{4}$ and three-dimensional Minkowski space. The warp-factor is induced by non-trivial background fluxes for the M-theory four-form field strength, but crucially contains contributions from higher-curvature terms of the eleven-dimensional action. Global consistency requires these to be included for compact internal manifolds. The required higher-curvature terms are suppressed by an additional factor of $\alpha^{2} \propto \ell_{M}^{6}$. Within an $\alpha$-expansion we were able to consistently include all required higher-derivative terms when determining the background solution and performing the dimensional reduction. The resulting three-dimensional action was already presented in [7]. In this analysis we have so far included the deformations of the Kähler structure of the geometry and the vector modes from the M-theory three-form. Due to the increasing computational complexity we performed the derivations only to leading non-trivial order in the fluctuations of the Kähler structure. Nonetheless we were able to identify key features of the effective action associated with warping and higher-derivative 
terms. One focus of this work was on demonstrating compatibility with the structure of a three-dimensional $\mathcal{N}=2$ supergravity theory.

As a first result we have shown that the scalar potential is only induced by background fluxes. Interestingly the back-reaction at order $\alpha^{2}$ on the background solution was crucial to establish this result. Dimensionally reducing the relevant higher-curvature terms (2.21) on a Calabi-Yau fourfold we have found a scalar potential for the Kähler deformations purely induced by geometry. However, these terms cancel precisely with term from the back-reacted metric and led to a confirmation of the flux-induced result of [19]. We stress that this cancellation arises only due to the non-trivial eleven-dimensional Weyl rescaling involving the scalar $Z$ cubic in the Riemann curvature. This rescaling can be also performed in eleven dimensions to modify the starting action before the dimensional reduction.

The main focus of this work was the study of the dimensionally reduced action with respect to three-dimensional supersymmetry. We used the result of [7] and determined the form of the $\mathcal{N}=2$ Kähler potential $K$ and complex coordinates $T_{i}$. The findings of [7] were only at lowest order in the fluctuations $\delta v^{i}$, which suggested that we may first determine $K, T_{i}$ as a $\delta v^{i}$-expansion. Already in this evaluation the main complication of the dimensional reductions at higher-derivative level became apparent. At lowest order in $\alpha$ it is straightforward to take the fluctuated result for $K, T_{i}$ and 'integrate' it into a closed expression depending on the Kähler form. It is well-known that in this case the Kähler metric, the Kähler potential, and the coordinates $T_{i}$ only depend on topological information, namely the intersection numbers, of the manifold $Y_{4}$. At order $\alpha^{2}$, however, the result of the dimensional reduction contains couplings that are not topological and 'integrating' these couplings into closed expressions turned out to be challenging.

As a first example, we found that the three-dimensional action contains kinetic terms involving the warp-factor $W$ in the integral $\int_{Y_{4}} W \omega_{i} \wedge \omega_{j} \wedge J \wedge J$. This integral is not topological and depends on the actual forms $\omega_{i}$ chosen to give its expression. Throughout this work $\omega_{i}$ were the $h^{1,1}\left(Y_{4}\right)$ harmonic representatives in the lowest order Ricci-flat metric. We have argued that there is at least no obvious integral over $Y_{4}$ with only one free-index $\omega_{i}$ that yields the above integral upon taking a $v^{i}$ derivative. Remarkably, at least for the warp-factor terms, one can find a way around this problem by defining $T_{i}$ to be given by integrals over divisors $D_{i}$. Our key observation was that the $v^{i}$-derivatives of the warpfactor equation allows us to write $\partial_{j} T_{i}$ as $Y_{4}$-integrals. Furthermore, this $v^{j}$-variation of $T_{i}$ was argued to only depend on the homology class of the divisor $D_{i}$ and not the precise representative. One might reinterpret this as a statement that one now has to consider not only topological integrals, but integrals that are 'semi-topological' up to usage of the warpfactor equation. We believe that a deeper understanding of this fact will shed more light onto the proper treatment of effective actions computed in warped string compactifications. Importantly, since the warp-factor equation also contains higher-curvature terms, we have shown that the terms including the warp-factor and the higher-derivative terms cannot be analysed independently.

The analysis of the higher-derivative terms turned out to be even more involved. Similar to the warp-factor terms we encountered after dimensional reduction non-topological metric-dependent integrals, such as $\int_{Y_{4}} Z \omega_{i} \wedge \omega_{j} \wedge J \wedge J$ and $\int_{Y_{4}} \omega_{i}^{\bar{n} m} \omega_{j}^{\bar{s} r} Z_{m \bar{n} r \bar{s}} * 1$, that 
should arise from a Kähler potential. As a $\delta v^{i}$ expansion we have shown compatibility of this metric with the existence of a Kähler potential and complex coordinates $T_{i}$. However, fully integrating these expressions to all orders in the fluctuations turned out to be challenging. We proposed an expression for $K$ and $T_{i}$ in (3.25) and (3.27). Remarkably, the form (3.27) of $T_{i}$ is severely constraint by the warp-factor equation. It does, however, contain the six-form $F_{6}$, which is constrained by (3.28) and therefore contains information about the non-harmonicity of the fourth Chern-form $c_{4}$ in the Ricci-flat metric. The form $F_{6}$ should capture the higher-derivative terms in the three-dimensional action, but we were not able to give its full definition including its moduli dependence. The equation (3.28) allows for arbitrary shifts of $F_{6}$ with harmonic six-forms. Such shifts will in general modify $T_{i}$ and cannot be a symmetry of the system. By matching with the result of the dimensional reduction we have found that the definition of $F_{6}$ has to satisfy (3.38). These conditions constrain the harmonic part of $F_{6}$. It would be of crucial importance to give an independent definition of $F_{6}$ satisfying (3.28) and (3.38). Our findings suggest already that there is a lot of structure in the higher-derivative terms appearing in the effective theory.

An immediate extension of our analysis is the dimensional reduction to next order in the fluctuations $\delta v^{i}$, since it would help to further uncover the underlying higher-derivative structures. While all four-dimensional couplings at the leading order in the $\delta v^{i}$-fluctuations can be written to depend only on the higher-curvature quantity $Z_{m \bar{m} n \bar{n}}$ a preliminary analysis to the next order suggests that other higher-curvature couplings are relevant. It would therefore be interesting to classify the relevant building blocks in the future.

Let us close by mentioning a further direction that deserves investigation. The presented results only deal with a three-dimensional $\mathcal{N}=2$ effective action. A natural next step is to also investigate the F-theory uplift of our findings to a four-dimensional $\mathcal{N}=1$ theory. This requires for the internal manifold $Y_{4}$ to be elliptically fibered. Shirking the fiber volume then yields the appearance of an extra circle. This limit is clearly complicated and requires the inclusion of further states that are not present in supergravity. However, applied to our reduction results the complications are even more immediate. In fact, it is an interesting open question how non-topological terms, for example including the warp-factor, are lifted to four space-time dimensions.

\section{Acknowledgments}

We would like to thank Iñaki García-Etxebarria, Daniel Junghans, Luca Martucci, Ruben Minasian, Diego Regalado, and Raffaele Savelli for useful discussions. This work was supported by a grant of the Max Planck Society.

Open Access. This article is distributed under the terms of the Creative Commons Attribution License (CC-BY 4.0), which permits any use, distribution and reproduction in any medium, provided the original author(s) and source are credited. 


\section{References}

[1] C. Vafa, Evidence for F-theory, Nucl. Phys. B 469 (1996) 403 [hep-th/9602022] [InSPIRE].

[2] K. Becker and M. Becker, M theory on eight manifolds, Nucl. Phys. B 477 (1996) 155 [hep-th/9605053] [INSPIRE].

[3] K. Becker and M. Becker, Supersymmetry breaking, M-theory and fluxes, JHEP 07 (2001) 038 [hep-th/0107044] [INSPIRE].

[4] T.W. Grimm, T.G. Pugh and M. Weissenbacher, On M-theory fourfold vacua with higher curvature terms, Phys. Lett. B 743 (2015) 284 [arXiv:1408.5136] [INSPIRE].

[5] H. Lü, C.N. Pope, K.S. Stelle and P.K. Townsend, Supersymmetric deformations of $G_{2}$ manifolds from higher order corrections to string and M-theory, JHEP 10 (2004) 019 [hep-th/0312002] [INSPIRE].

[6] H. Lü, C.N. Pope, K.S. Stelle and P.K. Townsend, String and M-theory deformations of manifolds with special holonomy, JHEP 07 (2005) 075 [hep-th/0410176] [INSPIRE].

[7] T.W. Grimm, T.G. Pugh and M. Weissenbacher, The effective action of warped M-theory reductions with higher derivative terms. Part I, arXiv: 1412.5073 [INSPIRE].

[8] E. Cremmer, B. Julia and J. Scherk, Supergravity Theory in Eleven-Dimensions, Phys. Lett. B 76 (1978) 409 [INSPIRE].

[9] M.J. Duff, J.T. Liu and R. Minasian, Eleven-dimensional origin of string-string duality: A One loop test, Nucl. Phys. B 452 (1995) 261 [hep-th/9506126] [INSPIRE].

[10] M.B. Green and P. Vanhove, D instantons, strings and M-theory, Phys. Lett. B 408 (1997) 122 [hep-th/9704145] [INSPIRE].

[11] M.B. Green, M. Gutperle and P. Vanhove, One loop in eleven-dimensions, Phys. Lett. B 409 (1997) 177 [hep-th/9706175] [INSPIRE].

[12] E. Kiritsis and B. Pioline, On $R^{4}$ threshold corrections in IIB string theory and $(p, q)$-string instantons, Nucl. Phys. B 508 (1997) 509 [hep-th/9707018] [INSPIRE].

[13] J.G. Russo and A.A. Tseytlin, One loop four graviton amplitude in eleven-dimensional supergravity, Nucl. Phys. B 508 (1997) 245 [hep-th/9707134] [INSPIRE].

[14] I. Antoniadis, S. Ferrara, R. Minasian and K.S. Narain, $R^{4}$ couplings in $M$ and type-II theories on Calabi-Yau spaces, Nucl. Phys. B 507 (1997) 571 [hep-th/9707013] [INSPIRE].

[15] A.A. Tseytlin, $R^{4}$ terms in 11 dimensions and conformal anomaly of $(2,0)$ theory, Nucl. Phys. B 584 (2000) 233 [hep-th/0005072] [InSPIRE].

[16] J.T. Liu and R. Minasian, Higher-derivative couplings in string theory: dualities and the B-field, Nucl. Phys. B 874 (2013) 413 [arXiv:1304.3137] [InSPIRE].

[17] S. Katmadas and R. Minasian, $\mathcal{N}=2$ higher-derivative couplings from strings, JHEP 02 (2014) 093 [arXiv:1311.4797] [INSPIRE].

[18] M. Haack and J. Louis, Duality in heterotic vacua with four supercharges, Nucl. Phys. B 575 (2000) 107 [hep-th/9912181] [INSPIRE].

[19] M. Haack and J. Louis, M theory compactified on Calabi-Yau fourfolds with background flux, Phys. Lett. B 507 (2001) 296 [hep-th/0103068] [INSPIRE].

[20] F. Bonetti, T.W. Grimm and T.G. Pugh, Non-Supersymmetric F-theory Compactifications on $\operatorname{Spin}(7)$ Manifolds, JHEP 01 (2014) 112 [arXiv: 1307.5858] [INSPIRE]. 
[21] D. Prins and D. Tsimpis, Type IIA supergravity and M-theory on manifolds with SU(4) structure, Phys. Rev. D 89 (2014) 064030 [arXiv:1312.1692] [INSPIRE].

[22] D. Prins and D. Tsimpis, $3 d \mathcal{N}=1$ effective supergravity and $F$-theory from $M$-theory on fourfolds, JHEP 09 (2015) 107 [arXiv: 1506.07356] [INSPIRE].

[23] K. Dasgupta, G. Rajesh and S. Sethi, $M$ theory, orientifolds and G-flux, JHEP 08 (1999) 023 [hep-th/9908088] [INSPIRE].

[24] S.B. Giddings, S. Kachru and J. Polchinski, Hierarchies from fluxes in string compactifications, Phys. Rev. D 66 (2002) 106006 [hep-th/0105097] [INSPIRE].

[25] S.B. Giddings and A. Maharana, Dynamics of warped compactifications and the shape of the warped landscape, Phys. Rev. D 73 (2006) 126003 [hep-th/0507158] [INSPIRE].

[26] C.P. Burgess et al., Warped Supersymmetry Breaking, JHEP 04 (2008) 053 [hep-th/0610255] [INSPIRE].

[27] G. Shiu, G. Torroba, B. Underwood and M.R. Douglas, Dynamics of Warped Flux Compactifications, JHEP 06 (2008) 024 [arXiv:0803.3068] [INSPIRE].

[28] M.R. Douglas and G. Torroba, Kinetic terms in warped compactifications, JHEP 05 (2009) 013 [arXiv: 0805.3700] [INSPIRE].

[29] L. Martucci, On moduli and effective theory of $N=1$ warped flux compactifications, JHEP 05 (2009) 027 [arXiv:0902.4031] [INSPIRE].

[30] B. Underwood, A Breathing Mode for Warped Compactifications, Class. Quant. Grav. 28 (2011) 195013 [arXiv: 1009.4200] [INSPIRE].

[31] T.W. Grimm, D. Klevers and M. Poretschkin, Fluxes and Warping for Gauge Couplings in F-theory, JHEP 01 (2013) 023 [arXiv:1202.0285] [INSPIRE].

[32] A.R. Frey and J. Roberts, The Dimensional Reduction and Kähler Metric of Forms In Flux and Warping, JHEP 10 (2013) 021 [arXiv:1308.0323] [INSPIRE].

[33] L. Martucci, Warping the Kähler potential of F-theory/IIB flux compactifications, JHEP 03 (2015) 067 [arXiv:1411.2623] [INSPIRE].

[34] T.W. Grimm, R. Savelli and M. Weissenbacher, On $\alpha^{\prime}$ corrections in $N=1$ F-theory compactifications, Phys. Lett. B $\mathbf{7 2 5}$ (2013) 431 [arXiv:1303.3317] [INSPIRE].

[35] T.W. Grimm, J. Keitel, R. Savelli and M. Weissenbacher, From M-theory higher curvature terms to $\alpha^{\prime}$ corrections in F-theory, arXiv:1312.1376 [INSPIRE].

[36] D. Junghans and G. Shiu, Brane curvature corrections to the $\mathcal{N}=1$ type-II/F-theory effective action, JHEP 03 (2015) 107 [arXiv:1407.0019] [INSPIRE].

[37] D. Ciupke, J. Louis and A. Westphal, Higher-Derivative Supergravity and Moduli Stabilization, JHEP 10 (2015) 094 [arXiv: 1505.03092] [INSPIRE].

[38] R. Minasian, T.G. Pugh and R. Savelli, F-theory at order $\alpha^{\prime 3}$, JHEP 10 (2015) 050 [arXiv: 1506. 06756] [INSPIRE].

[39] E. Witten, Nonperturbative superpotentials in string theory, Nucl. Phys. B 474 (1996) 343 [hep-th/9604030] [INSPIRE].

[40] P. Griffiths and J. Harris, Principles of Algebraic Geometry, John Wiley and Sons, Inc. (1978).

[41] R. Bott and L.W. Tu, Differential Forms In Algebraic Topology, Springer-Verlag (1982). 
[42] E. Witten, Five-brane effective action in M-theory, J. Geom. Phys. 22 (1997) 103 [hep-th/9610234] [INSPIRE].

[43] D. Freed, J.A. Harvey, R. Minasian and G.W. Moore, Gravitational anomaly cancellation for M-theory five-branes, Adv. Theor. Math. Phys. 2 (1998) 601 [hep-th/9803205] [INSPIRE].

[44] K. Lechner, P.A. Marchetti and M. Tonin, Anomaly free effective action for the elementary M5-brane, Phys. Lett. B 524 (2002) 199 [hep-th/0107061] [INSPIRE]. 